\section{Greenhouse effect economics}

SIR-The recognition that the "social and economic issues are as important as the strictly technical" in relation to global warming (Nature 345, 473; 1990) is important. Economic analysis has, indeed, a number of contributions to make, of which two are particularly important. The first is to establish how much (if any) action should be initiated to limit global climate change. Second, economics can assist in identifying the most efficient (least cost) means of achieving any desired target for emission control.

John Maddox makes a valuable contribution by sketching the magnitude of the global " $\mathrm{CO}_{2}$ business". He argues that with fossil fuel consumption releasing approximately $25 \times 10^{12} \mathrm{~kg}$ of $\mathrm{CO}_{2}$ to the atmosphere annually, "there is no other commodity in world commerce routinely handled on such a scale".

But this raises a central aspect of the economics of global warming. Carbon dioxide is not a commodity, and it is not traded in world commerce (except in very limited quantities). In other words, carbon emissions constitute an externality to production processes - they generate real costs to society, but costs not borne by the emitters.

That $\mathrm{CO}_{2}$ emissions have a zero price is the nub of the problem. Economically sensible decisions about resource use in market economies (or in planned economies where planners use 'shadow prices') require the presence of correct price signals. That is why economists have for a long time recommended that externalities such as greenhouse gas emissions be taxed at their true marginal social cost, so that decision makers take account of the full consequences of their decisions.

Maddox argues, correctly, that the costs of abating emissions may be very high. But his figures fail to distinguish between marginal and average (or total) control costs. This distinction is critical in considering pollution control policy. It is argued that substitution of fossil fuels by other sources would be more expensive by a factor of three, with initial capital costs at least 10 times as large. This is misleading: the marginal cost of substitution can be very low for initial fuel-switching projects, although it will tend to rise fairly sharply as the extent of substitution increases.

So we should not view the problem in terms of totals. Certainly, replacing all fossil-fuel generated electricity by other sources will be hugely expensive, but substitution at the margin may be relatively cheap. Indeed, in the short term, the cost of emission control programmes through energy conservation and efficiency schemes may actually be negative; such projects would generate positive net

\section{present values}

We now need to focus research on quantification of the marginal cost schedule for emissions control, which is likely to have a shape like that in the figure.

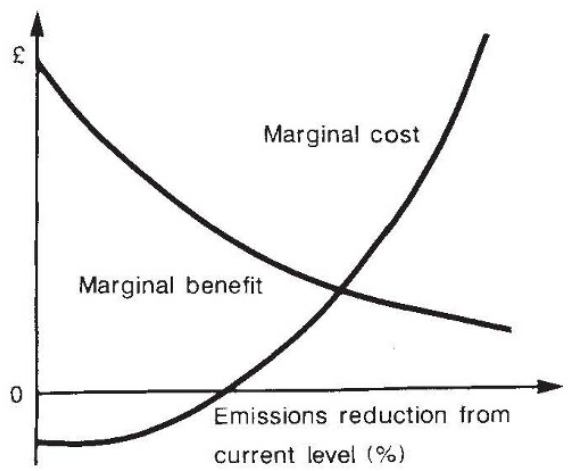

Optimal policy can then be identified if we can estimate the marginal benefits function (or, the benefit society derives in the form of averted global climate change from unit reductions in emissions). Equating marginal costs and benefits identifies the best target for emissions control. Alternatively, policy makers can use such a framework to ask how severe global warming damage would have to be to justify a particular level of intervention.

This is clearly a mammoth undertaking, but is not to be avoided for that reason. Several teams of economists have been working on this problem for a number of years, and the interested reader can look at the work of William Nordhaus (Yale University) for some 'ball park' estimates of policy targets derived in this way. Nordhaus ${ }^{1}$ estimates that about 14 per cent of greenhouse gas emissions can be reduced at extremely low cost. Above this, marginal costs rise sharply from about $\$ 38$ per ton $\mathrm{CO}_{2}$ at 25 per cent reduction levels to $\$ 119$ per ton for a 50 per cent reduction. Nordhaus examines three plausible marginal benefit functions (averted damage); for his low, medium and high versions, he finds the optimal targets to be greenhouse gas emission cuts of 10 per cent, 17 per cent and 50 per cent respectively.

These figures relate to optimal targets assuming the best (cheapest) option for reduction is taken at each step. The second contribution that economics can make is to guide policy makers on the appropriate policy instruments for realising least-cost control. I hope that Nature can contribute to this debate much as it has to our analysis of the "strictly technical" questions that it normally addresses.

ROGER PERMAN

Dept of Economics, University of Strathclyde, Glasgow G4 OLN, UK

1. Nordhaus, W.D. To Slow Or Not To Slow: The Economics of the Greenhouse Effect (in the press).
RSPCA policy

SIR-In your leading article "Escalation of animal rights war" (Nature 345, 647; 1990) you use a form of words bound to create ambiguity in the minds of readers.

The policy of the RSPCA, to prevent cruelty and promote kindness to animals by all lawful means, is crystal clear and always has been. Membership of the RSPCA is conditional upon adherence to that policy.

This society does not condone the breaking of the law and utterly condemns those extremists who, by their actions, place human life in danger.

Royal Society for the Prevention

of Cruelty to Animals,

Causeway, Horsham,

West Sussex RH12 1HG, UK

SIR - As a rational, intelligent person, I was offended by the total lack of balance in the leading article about animal rights.

Terrorist bombing is indefensible, and I abhor it, as do all my acquaintances concerned with advocacy for animals. However, the violent actions of a few do not invalidate sound ethics and reasoning.

'Speciesism' is a handy term for our continuing use of animals although there are no morally relevant differences to justify doing to them what we would not do to ourselves. It has been discussed by respected academic philosophers such as Peter Singer, Tom Regan, Bernard Rollin and M. A. Fox, who on reflection repudiated his own book The Case for Animal Experimentation in The Scientist (December 1986). What privileged insight entitles your author to dismiss it as "claptrap"?

The parallel drawn between anaesthesia and death evades the major issue of pain and distress inflicted on conscious animals in the laboratory. A reading of the scientific literature shows that there is no need for exaggeration: witness, for example, autotomy due to deliberately inflicted pain, described by Melzack (Exp. Neurol. 92, 713; 1986) and the administration of repeated inescapable shock by Anisman et al. (Pharmac. Biochem. Behav. 24, 323 \& 1151; 1986). Why did the author omit equal condemnation of the grossly exaggerated claims that animal research is responsible for every medical advance (though not for any of the failures)? Consider the examples of insulin and penicillin, cited in Robert Sharpe's book The Cruel Deception.

People with heart disease and ulcers (among others) may well benefit more from sound clinical investigations by physicians doing relevant and humane research than by trusting their health to results derived from animal models, as these may fail the test of extrapolation.

R. M. KESTEVEN

RRI Sydenham, Ontario KOH 2TO, Canada 